\title{
Design and Development of Vehicle Collision- Avoidance System Based on UWB Wireless Sensor Networks
}

\author{
Xu Liu' ${ }^{12}$ \\ Beijing General Research Institute of Mining and Metallurgy \\ Beijing Key Laboratory of Nonferrous Intelligent Mining Technology \\ Beijing, 100260,China \\ E-mail: Iiuxu@bgrimm.com \\ Feng Jin ${ }^{\mathbf{a}}$, Xiao Lv' ${ }^{\mathbf{b}}$, Yuan Sheng Zhang ${ }^{\mathbf{c}}$, \\ Beijing General Research Institute of Mining and Metallurgy \\ Beijing Key Laboratory of Nonferrous Intelligent Mining Technology \\ Beijing, 100000, China \\ E-mail: ${ }^{a} j$ infeng@bgrimm . com ; ${ }^{b}$ vxiaolbgrimm. com ${ }^{\circ}{ }^{c}$ hangyuansheng@bgrimm. com
}

\section{Da Zhang}

Beijing General Research Institute of Mining and Metallurgy

Beijing Key Laboratory of Nonferrous Intelligent Mining Technology

Beijing, 100000,China

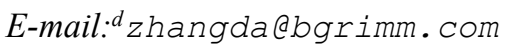

\begin{abstract}
In order to solve the personal injury accidents when the vehicle drives in the mine, this paper designs and developments an vehicle collision-avoidance system based on UWB Wireless Sensor Networks which can solve the personal injury accidents. The UWB is an emerging technology designed at short range and high transmission rate which can offer a fine resolution to evaluate distance. This paper presents the vehicle collision-avoidance system composition which consists of both the hardware composition and software design. It also presents the principle of ranging algorithm which is used to implement the function of vehicle collision-avoidance. The vehicle collision-avoidance system has been tested in the mine and the personal injury accidents have greatly decreased. Therefore, the system will have a fine application prospects in mine.
\end{abstract}

CENet 2017

July 22-23, 2017

Shanghai, China

\footnotetext{
${ }^{1}$ Speaker

${ }^{2}$ This research was supported by International S\&T Cooperation Program of China (Grant 2015DFA60330).
} 


\section{Introduction}

Trackless conveying is one of the most important conveying ore modes in metallic and non-metallic mines in China. As the ore resources gradually becoming rare, the depth of underground mining increasing and geological conditions deteriorating, the traditional trackless conveying system is generally facing with the problems such as terrible operation environment, onerous transport tasks and backward management techniques, etc. Taking the large-scale transportation system, as lacking of vehicle collision-avoidance system and safety pre-warning system which cause accidents as as example, both the production and conveying efficiency are affected. The existing security is also at risk.

Therefore, design and development of the vehicle collision-avoidance system in the metal and non-metal mines underground is an urgent task. This paper adopts UWB network protocol to develop vehicle collision-avoidance system, which enhances the level of a safe and efficient operation underground trackless transport system. The vehicle collision-avoidance system has the urgent demand and is of great significance.

The UWB technology is adopted in this paper to realize the vehicle collision-avoidance system. At present, many companies have introduced UWB chip which is widely used in wireless communications, networks, radar systems, image processing and positioning systems. It provides a good foundation of hardware for our system design.

\section{The UWB Protocol}

The UWB pulse radio technology has a rapid development in recent years and attracts much attention from the industrial and academic world. It has numerous advantages over the sinusoidal carrier-based communication technology, and enables effective and reasonable transmission in the wireless sensor network[1,2]. The UWB is an emerging technology designed at short range and high transmission rate[3,4]. Besides, the UWB can offer a fine resolution when it comes to evaluate distance or location[5].

UWB network protocol not only refers to DS-CDMA or MB-OFDM, but also includes the network protocol model as shown in Figure 1.

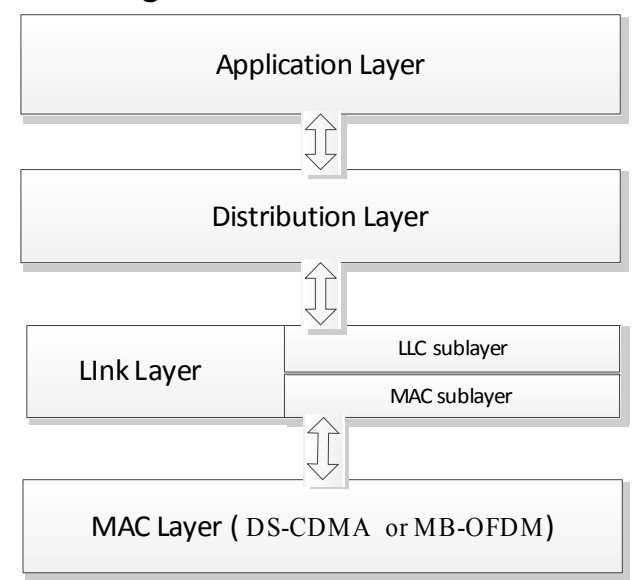

Figure 1: Structure of UWB Protocol

The application layer protocol includes DLAN compliant standard etc. The signal transmitted from application Layer converges in the distribution Layer. The signal is converted into the same by distribution Layer to transmit MAC Layer, and then the signal is transmited by the MAC Layer. Link Layer is composed of LLC sublayer and MAC sublayer. MAC sublayer 
realizes media access control, synchronize, power control and authenticated encryption. MAC sublayer protocol mainly refers to DS-CDMA or MB-OFDM which locates in the bottom of the frame, and it brings about physical frame, scrambling, coding, interweaving, modulation functions etc.[6-7].

The communication mode of the UWB network is based on the channel sharing. The media access control technology of the MAC sublayer comes from limited Lan that is called Carrier Sense Multiple Access with Collision Detection (CSMA/CD). The Wireless Local Area Network 802.11 defines CSMA/CD as a new protocol to improve the uncontested transmission.

\section{The Principle of Ranging Algorithm}

The vehicle collision-avoidance system uses the ranging algorithm to calculate the range as follows: one end acts as a Tag, periodically initiating a range measurement, while the other end acts as an Anchor listening and responding to the Tag and calculating the range. The Tag sends a Poll message addressed to the target Anchor and notes the send time, $\mathrm{T}_{\mathrm{SP}}$ for short. The Tag listens to the Response message. If no response arrives after some period the Tag will time out and send out the poll again. The Anchor listens to a Poll message addressed to it. When the Anchor receives a poll, it notes the received time $T_{R P}$ for short, and sends a Response message back to the Tag, noting its sending time $\mathrm{T}_{\mathrm{SR}}$ for short. When the Tag receives the Response message, it notes the receiving time $T_{R R}$ and sets the future sending time of the Final response message $\mathrm{T}_{\mathrm{SF}}$ for short, it embeds this time in the message before initiating the delayed sending of the Final message to the Anchor. The Anchor which receives this Final response message now has enough information to work out the range. In the principle of ranging algorithm the Anchor sends a ranging report of the calculated range to the Tag. Figure 2 shows this exchange and gives the formula used in the calculation of the range.

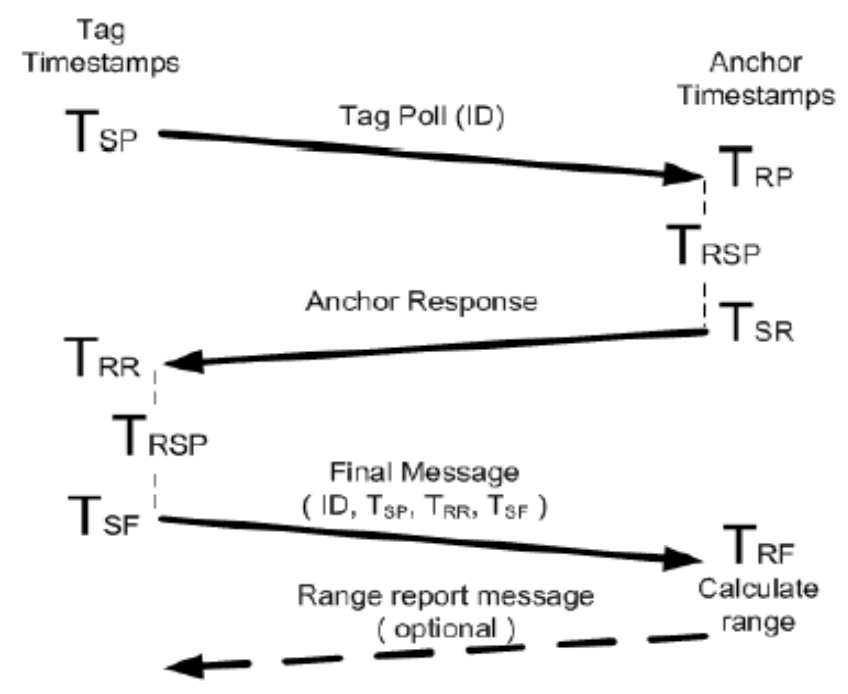

Figure 2: Exchange of the Tag and the Anchor

The first Round Trip Delay time of the Tag (

$$
\mathrm{T}_{\mathrm{TRT}}=\mathrm{T}_{\mathrm{RR}}-\mathrm{T}_{\mathrm{SP}}
$$

The first Round Trip Delay of Anchor（short for $\mathrm{T}_{\mathrm{ART}}$ ）:

$\mathrm{T}_{\mathrm{ART}}=$

$$
\mathrm{T}_{\mathrm{RF}}-\mathrm{T}_{\mathrm{SR}}
$$


After receiving the final message, the Anchor knows all the timestamps, so it can:

(a) remove its response time: $\left(\mathrm{T}_{\mathrm{SR}}-\mathrm{T}_{\mathrm{RP}}\right)$ from the $\mathrm{T}_{\mathrm{TRT}}$ of the Tag.

(b) remove the Tags' response time: $\left(\mathrm{T}_{\mathrm{SF}}-\mathrm{T}_{\mathrm{RR}}\right)$ from the $\mathrm{T}_{\mathrm{ART}}$ of the Anchor, and give antenna to antenna round trip times

The Anchor then averages those two resultant round trip times to remove the effects of each end's clock frequency differences, and divides by 2 to get a one-way trip time.

The Time of Flight shows as follows:

$$
T O F=\left(2 * \mathrm{~T}_{\mathrm{RR}}-\mathrm{T}_{\mathrm{SP}}-2 * \mathrm{~T}_{\mathrm{SR}}+\mathrm{T}_{\mathrm{RP}}+\mathrm{T}_{\mathrm{RF}}-\mathrm{T}_{\mathrm{SF}}\right) / 4
$$

And then we can calculate the distance between the two devices by multiplying $\mathrm{c}$ which is the speed of light.

$$
\text { Distance }=\mathrm{C} * \mathrm{TOF}
$$

In order to measure more accurately the distance from the person to the vehicle, we use four Anchor devices at each corner of the vehicle, as shown in Figure 3. The system can get four distances, and we accept the shortest distance as the distance from the person to the vehicle. When the person goes into the dangerous zone, the system can provide an alarm function to alert the person and the driver to leave far from the vehicle or to stop the vehicle.

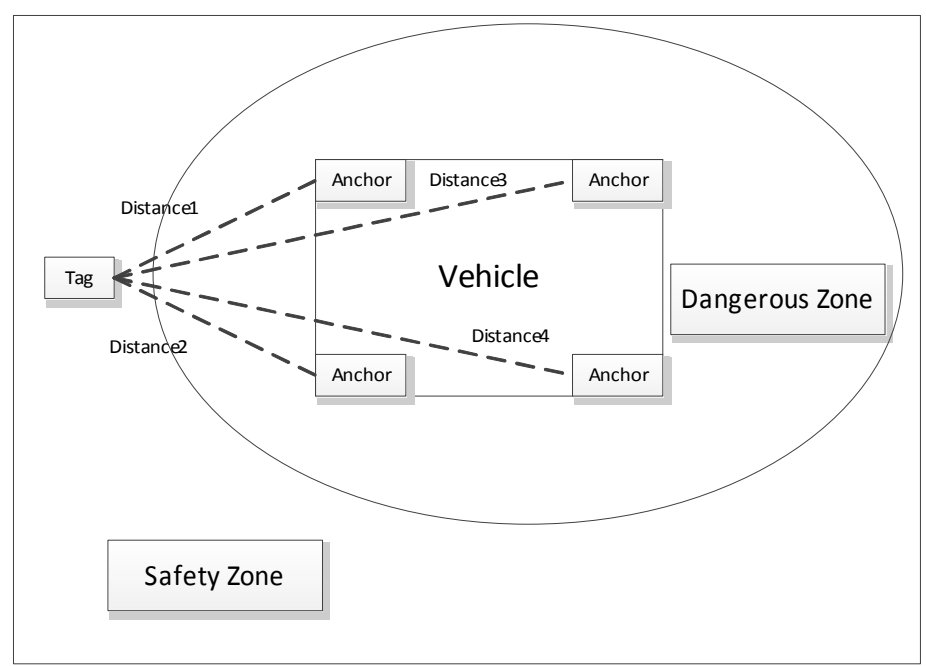

Figure 3: The Range of the Person and the Vehicle

\section{The Hardware of the System}

The Anchor and Tag node is the key of the hardware design. The Anchor node mainly consists of the processor module, the wireless transceiver module and the embedded software. Anchor node is used STM32F105RCT6 as MCU, and the SPI to connect and control the DW1000 wireless transceiver chip. The Anchor node is used to store the MAC address, IP address and the server IP address etc. with the block integrated EEPROM chip. The Anchor node is designed with Ethernet interface which is used to upload data and receive data, as shown in Figure 4. 


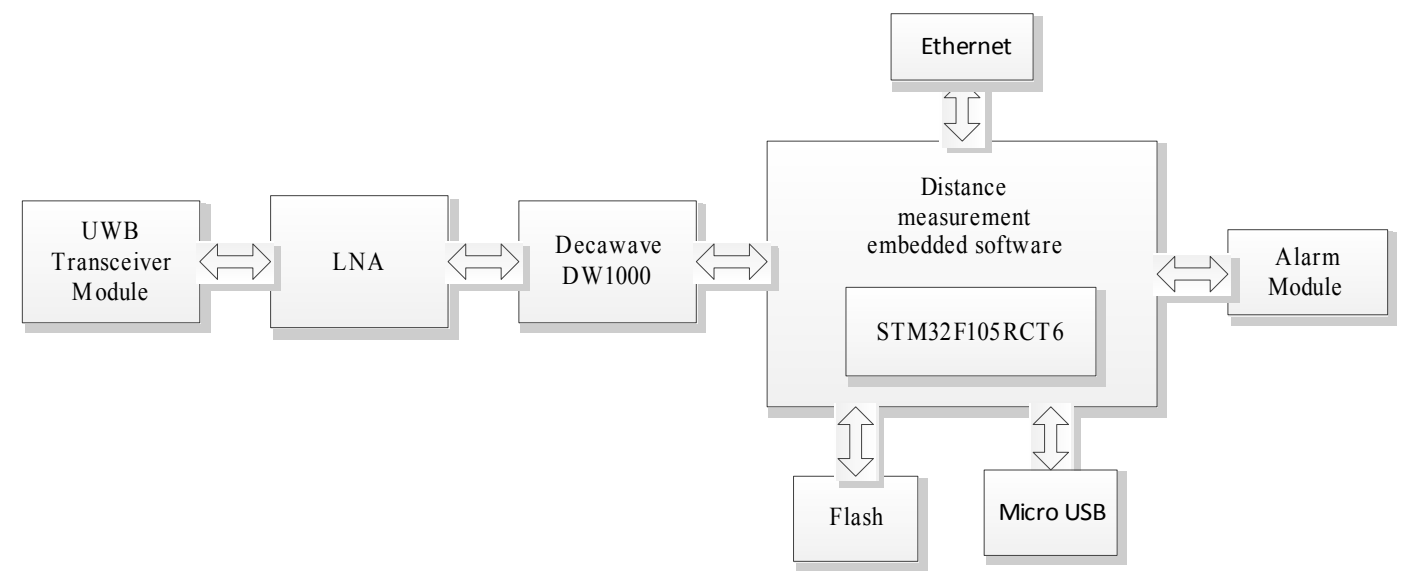

Figure 4: The Hardware of the System

\subsection{The MCU Module}

This paper chooses STM32F105RCT6 which is produced by STMicroelectronics ${ }^{[8]}$ as MCU. The chip incorporates a variety of high performance industrial standard interfaces. The MCU is based on the ARM Cortex-M3 kernel and its maximum frequency is $72 \mathrm{MHz}$. It has 64 to 256 Kbytes of Flash memory and up to 64 Kbytes of general-purpose SRAM. The chip supports the peripherals as follows: timers, SPIs, I2Cs, USARTs, and USB2.0 OTG, a full speed connection that supports power supply.

\subsection{UWB Module}

The UWB module adopts the DW1000 wireless transceiver chip which is produced by the Deca-Wave company. The DW1000 is a fully integrated single chip Ultra Wideband (UWB) low-power low-cost transceiver IC compliant to IEEE802.15.4-2011 which can be used in 2way ranging or TDOA location systems to locate assets to a precision of $10 \mathrm{~cm}$. It also supports data transfer at rates up to $6.8 \mathrm{Mbps}$. The UWB module supports $6 \mathrm{RF}$ bands from $3.5 \mathrm{GHz}$ to $6.5 \mathrm{GHz}$ and low power consumption with sleep mode current $1 \mathrm{uA}$. Meanwhile, it has the complete MAC support software and provides SPI interface which connects to the host processor[9].

\subsection{Design of the SPI Interface}

The DW1000 host communications interface is a slave-only SPI, compliant with the industry protocol. The host system must include a master SPI bus controller to communicate with DW1000. Each SPI transaction starts with a one to three octet transaction header followed by a variable number of octets making up the transaction data. The number of data bytes allowed in an SPI transfer is not limited. Physically, the SPI interface is full duplex in that every transaction shifts bits both into an out of the DW1000. Logically however, each transaction is either reading data from the DW1000 or writing data to it.

The SPI interface is designed with 4 lines: SCLK indicates the main device, STM32 which generates a clock signal to complete the control of the communication. MOIS represents the STM32 outputting data and the DW1000 receiving the data. MIOS stands for DW1000 outputting Data and STM32 receiving data. CS1/CS2 indicates the enabling signal of DW1000 which is controlled by STM32. Details are as shown in Figure 5. 


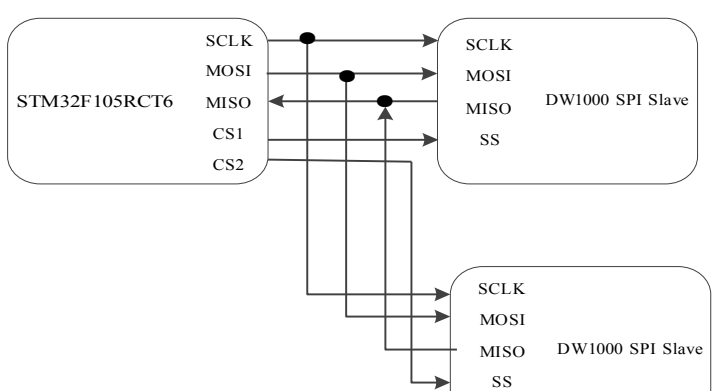

Figure 5: Design of the SPI Interface

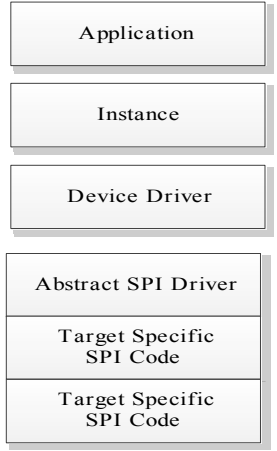

Figure 6: The Software of the System

\section{The Software of the System}

\subsection{The Layered Structure of the Software}

Figure 6 below shows the layered structure of the software with the main files associated with each layer and a brief description of the functionality provided at that layer. In the Application layer which runs the instance code, display results, provide interface for user control and configuration. In the Instance Layer, it provides the simple state machine to exchange messages to figure out the distance between the two units. The Device Driver Layer provides specific code to control DW1000 device functionality. The Abstract SPI Driver Layer realizes the generic PI function which should be easily portable to SPI of STM32F105RCT6. The Target Specific SPI Code Layer achieves reading and writing via the SPI of STM32F105RCT6. The physical SPI interface enables SPI wires to connect to SPI port on DW1000. Details are as shown in Figure 6.

\subsection{The Interrupt Handling of the DW1000}

This paper focuses on the research of the interrupt handling of the DW1000. Figure 7 shows how the DW1000 interrupts are processed by the microcontroller system. Once the interrupt is active the microcontroller's target specific interrupt handler for that interrupt line should get called, which in turn calls for the DW1000 device driver's interrupt handler service routine, the dwt_isr() API function, which processes the event that triggered the interrupt. 


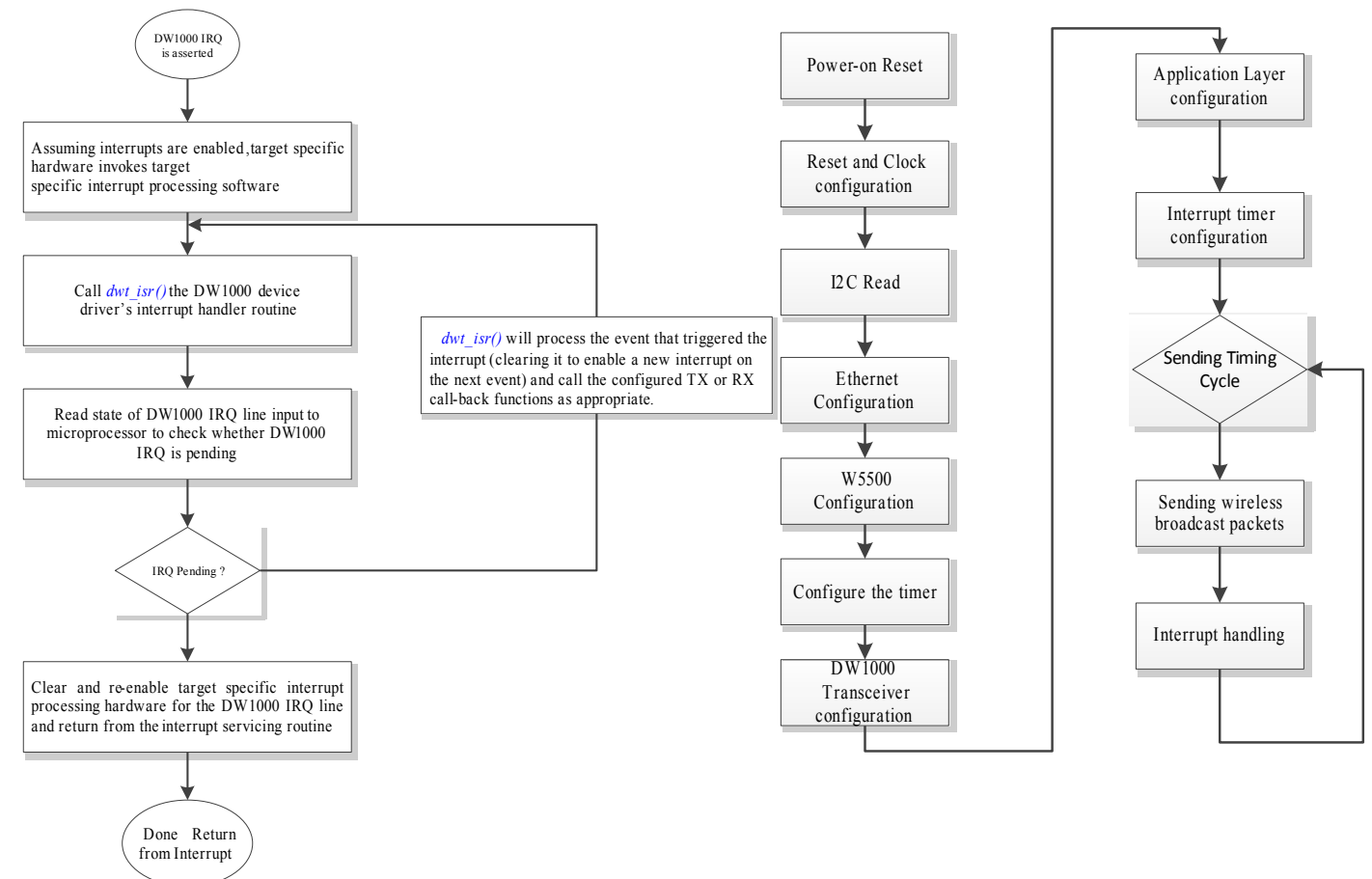

Figure 7: Interrupt Handling

Figure 8: The Flow Chart of the Sofware

\subsection{Software Flow of the System}

The embedded software adopts the serial processing to realize the functions such as STM32 resetting and clocking, the I2C configuration reading module, the Ethernet module, the dynamic IP access module, the UDP W5500 port initialization module, the timer, the DecaWave configuration module, the DW1000 transceiver configuration module, the upper application initialization module, the sending radio data package module and the interrupt handler. When the wireless transceiver chip receives or sends a data frame, it will send the interrupt request to the MCU. The interrupt program mainly produces the interrupt type, judges the correctness of the interrupt position and reads the data receiving interrupt source address. The received data frame reads the TOF information and the information packet, as shown in Figure 8.

The Anchor and Tag computes the distance, and then judges whether the distance is safe. If the distance is not safe, the system will provide the alarm function to alert the person and the driver to leave far from the vehicle or to stop the vehicle.

\section{Test of the System}

The vehicle collision-avoidance system is tested in a mine. The system can offer effective reminder and warning functions, especially for night driving or driving in the crooked tunnel. The security of the personnel in the mining area has been greatly improved.

\section{Conclusion}

This paper introduces an vehicle collision-avoidance system based on UWB network protocol. It presents the system composition, including the hardware composition and the software design. Through the vehicle collision-avoidance system, the efficiency of safety is greatly improved. In a word, the vehicle collision-avoidance system will have fine application prospects in the mine. 


\section{References}

[1]I.F. Akyildiz.A Survey on Sensor Networks [J]. IEEE Communication Magazine, 2002, (8): 102114.

[2]W.W. WU. UWB-Based Wireless Sensor Network. 《Zte Telecommunications》, 2005 (3) :16-20.

[3] X. J. Wang, L. Liu, F. Ye, J. Y. Ren, B. Hu, “A Novel Synchronizer for OFDM-based UWB System on New Preamble Design", the 18th Annual IEEE International Symposium on Personal, Indoor and Mobile Radio Communications (PIMRC'07), Greece, 2007, pp. 1-5.

[4] R. Kraemer, M. Katz. Short -range wireless communications: emerging technologies and applications USA: Wiley,2009.

[5] A. Chehri,P. Fortier,P. M. Tardif. UWB-Based Sensor Networks for Localizationin Mining Environments. Ad-Hoc Networks,2008( 5) : $179-188$.

[6]Y.P. Huang ,X.R. You,Channel estimation algorithm for TH-PPM UWB systems based on channel sparsity. Journal of Chongqing University of Posts \& Telecommunications.2010,22(1):2326

[7]X. N. Gong, X.M. Zhang. Realization of Automotive Anti-collision Radar Based on UWB. MICROCOMPUTER \& ITS APPLICATIONS.2004(9):18-20.

[8]Y. X. Lin. Ultrasonic Ranging Applied in Design and Analysis of Automotive Anti-collision Radar. AUTO ELECTRIC PARTS.2006, (12).

[9]X. Li, D. F. Zhao, R. Xue. MAC protocols based on UWB network.Journal of Heilongjiang Institute of Science \& Technology.2005,15(5):266-268. 